\title{
VERAPAMILO EN CONJUNTO CON LAS PRENDAS DE COMPRESIÓN EN EL TRATAMIENTO DE LA CICATRIZ PATOLÓGICA CAUSADA POR QUEMADURA*
}

\author{
Drs. Guillermo Ramos-Gallardo ${ }^{1}$, Ariel Miranda A. ${ }^{1}$, Teresa Chávez S. ${ }^{1}$, \\ Leonel García B. ${ }^{1}$, David Medina P. ${ }^{1}$, Sandra Figueroa J. ${ }^{1}$, \\ Leticia Chávez-Dagostino ${ }^{1}$, Rebeca Valdez-López ${ }^{1}$ \\ 1 Unidad de Atención al Niño Quemado, Nuevo Hospital Civil de Guadalajara "Juan I. Menchaca". \\ México.
}

\begin{abstract}
Verapamil in conjunction with pressure therapy in the treatment of pathologic scar due burn injury

Introduction: Keloids and hypertrophic scars are dermal fibro-proliferative disorders unique to humans. Their treatment is a true challenge with multiple options, but not all the time with good results. Unfortunately this problem is not uncommon in patients with history of burn injury. Aim: To evaluate use of verapamil and pressure garments in patients with hypertrophic or keloid scar caused by burn injury. Methods: We included patients with a hypertrophic or keloid scar caused by burn injury that report non-response to treatment with pressure garment. The pathologic scars were evaluated by serial photographic records, Vancouver and Posas scales. The scales of Vancouver and Posas were compared with t Student. Results: We included 13 scars in 11 patients. Four scars were located in the legs, 4 in the arms, 4 in the face-neck and 1 in the abdomen. The dose of verapamil was calculated $0.03 \mathrm{mg}$ per $\mathrm{kg}$. Injections were scheduled every 7 to 10 days until complete 6 sessions. Taking in count Posas scale, patients referred improvement in pigmentation (0.01), thickness $(0.005)$, pliability (0.01), pruritus (0.003) and irregular surface $(0.004)$. In the Vancouver scale the observers mentioned improvement in elevation (0.008), pigmentation (0.014), vascularity $(0.022)$ and flexibility $(0.014)$. No adverse effects were found in verapamil injection. Conclusion: Verapamil was useful in conjunction with pressure garment to improve the condition of the keloid and hypertrophic scar caused by burn.
\end{abstract}

Key words: Keloid, verapamil, pressure therapy.

\section{Resumen}

Introducción: La cicatriz queloide o la cicatriz hipertrófica son desórdenes fibro-proliferativos únicos de los humanos, cuyo tratamiento representa un reto en donde existen pocas opciones con buenos resultados. Objetivo: Evaluar el uso de verapamilo y las prendas de compresión en pacientes con cicatrización patológica como consecuencia de quemadura. Material y Método: Incluimos pacientes con cicatrización patológica, ya sea queloide o hipertrófica, causada por quemadura que mencionaron no haber tenido beneficio con el uso

*Recibido el 17 de noviembre de 2014 y aceptado para publicación el 25 de mayo de 2015.

Los autores no refieren conflictos de interés.

Correspondencia: Dr. Guillermo Ramos-Gallardo guiyermoramos@hotmail.com 
de prendas de compresión. La cicatriz fue evaluada con fotografías seriadas, escala de Vancouver y Posas. Los resultados fueron comparados con la prueba de $t$ de Student. Resultados: Incluimos 13 cicatrices en 11 pacientes. La localización de las cicatrices fue en brazos 4, piernas 4, cara y cuello 1, y abdomen 1. La dosis de verapamilo se calculó a $0,03 \mathrm{mg}$ por $\mathrm{kg}$. Las inyecciones se aplicaron intralesionales y se administraron cada 7 a 10 días hasta completar 6 sesiones. Encontramos mejoría en los siguientes parámetros de la escala de Posas: pigmentación $(0,01)$, pliabilidad $(0,01)$, endurecimiento o grosor $(0,005)$, prurito $(0,003)$ e irregular $(0,004)$. En la escala de Vancouver elevación $(0,008)$, pigmentación $(0,014)$, vascularidad $(0,022)$ y flexibilidad $(0,014)$. No encontramos efectos adversos con la administración de verapamilo. Conclusión: El verapamilo fue útil en conjunto con las prendas de compresión para mejorar las condiciones de la cicatriz queloide e hipertrófica causadas por lesiones por quemadura.

Palabras clave: Queloide, verapamilo, prendas de compresión.

\section{Introducción}

El proceso patológico que incluye las cicatrices hipertróficas o queloides son un problema de difícil manejo en pacientes con antecedentes de quemaduras. Extensas áreas se ven afectadas causando limitación en el movimiento y la función. Una opción útil de tratamiento es la terapia de presión, especialmente la utilizada con prendas, herramientas con un amplio uso en América del Norte y Europa ${ }^{1-3}$. Los pacientes con secuelas por quemaduras deben ser seguidos por un equipo multidisciplinario, donde muchos especialistas están involucrados con el fin de iniciar la rehabilitación e identificar alguna limitación en la función o el movimiento.

Por desgracia, durante el proceso de maduración de la cicatriz, además de las diversas medidas y esfuerzos, el problema continúa limitando la calidad de vida de los pacientes ${ }^{2,3}$.

Proponemos en estos pacientes utilizar verapamilo intralesional en combinación con terapia de presión, prendas, ya que el verapamilo ha demostrado ser una herramienta útil en el tratamiento de la cicatrización patológica, se ha reportado en la literatura cambios en la forma de los fibroblastos y la disminución de interleucina 6 así como otros marcadores $^{4-6}$. Si bien el proceso de cicatrización se completa en un tiempo de 12 meses decidimos usar verapamilo en un tiempo menor a los 12 meses con la intención de disminuir el tiempo de maduración de la cicatriz y mejorar la función y calidad de vida de nuestros pacientes. Nosotros usamos las prendas de compresión de manera rutinaria como lo propone el grupo de Seatlle encabezado por Gibran ${ }^{3}$. o existen informes en población mexicana acerca de este medicamento en pacientes con problemas de cicatrización por secuela de quemadura.

\section{Métodos}

Realizamos un estudio prospectivo que se llevó a cabo en pacientes con cicatriz queloide o hipertrófica a partir de enero de 2014 a junio de 2014.
Se incluyeron pacientes con diagnóstico reciente de lesión por quemadura (menos de 12 meses) que presentaron como secuela cicatriz hipertrófica o queloide en tratamiento con prendas de compresión que no tuvieron cambio o mejoría en la escala de Posas o Vancouver en dos visitas consecutivas con un intervalo de tiempo de un mes.

Los casos fueron documentados con imágenes de la cicatriz patológica (hipertrófica o queloide) antes y al final del tratamiento con verapamilo intralesional. Las escalas Posas y Vancouver fueron evaluadas antes y al primer mes después de terminar el tratamiento con verapamilo intralesional. Se inyectó verapamilo intralesional dosis $0,03 \mathrm{mg}$ por $\mathrm{kg}$ de peso cada 7 a 10 días hasta completar 6 sesiones con el objetivo de acelerar el proceso de maduración propio de la cicatriz. Los resultados de las escalas Posas y Vancouver se compararon con la prueba $t$ de Student.

El proyecto de investigación fue aprobado por el Comité de Ética del Nuevo Hospital Civil de Guadalajara Juan I. Menchaca (número: 1376-14).

\section{Resultados}

Se evaluaron 126 pacientes con historia reciente de lesiones por quemaduras en seis meses a partir de enero de 2014 y junio de 2014. De ellos 45 pacientes $(35 \%)$ utilizaron la terapia de presión (Cicamed $\left.{ }^{\circledR}\right)$. La presión que proporcionaron las prendas sobre las cicatrices fue de $25 \mathrm{~mm} / \mathrm{Hg}$. En 11 pacientes $(8,7 \%)$ no se encontró cambio o mejoría en la escala de Posas y Vancouver en dos visitas consecutivas con un lapso de un mes. Se administró verapamilo intralesional en 13 cicatrices de estos pacientes.

La Tabla 1 muestra paciente, ubicación y tipo de cicatriz evaluados. Figuras 1 y 2 muestran ejemplo de las cicatrices en el brazo derecho antes y después del tratamiento con verapamilo. Figuras 3 y 4 muestran ejemplo de la cicatriz en la cara y el cuello antes y después del tratamiento con verapamilo.

El medicamento se administró cada 7 a 10 días 
Tabla 1. Pacientes, localización, tamaño de la cicatriz, causa, tiempo de evolución

\begin{tabular}{|llllc|}
\hline Paciente & Localización & Tamaño de la cicatriz & Causa & Tiempo de Evolución \\
Masculino, 7 años & Cara & $6 \mathrm{~cm}$ por $1,5 \mathrm{~cm}$ & Quemadura, fuego & 8 meses \\
Masculino, 7 años & Cara & $3 \mathrm{~cm}$ por $2 \mathrm{~cm}$ & Quemadura, fuego & 8 meses \\
\hline Masculino, 15 años & Cuello & $5 \mathrm{~cm}$ por $1 \mathrm{~cm}$ & Quemadura, fuego & 10 meses \\
Femenino, 1 años & Brazo derecho & $8 \mathrm{~cm}$ por $2 \mathrm{~cm}$ & Escaldadura & 3 meses \\
\hline Femenino, 1 año & Brazo izquierdo & $12 \mathrm{~cm}$ por $4 \mathrm{~cm}$ & Escaldadura & 10 meses \\
\hline Femenino, 6 años & Brazo derecho & $2 \mathrm{~cm}$ por $5 \mathrm{~cm}$ & Escaldadura & 10 meses \\
\hline Femenino, 6 años & Abdomen & $8 \mathrm{~cm}$ por $1 \mathrm{~cm}$ & Escaldadura & 10 meses \\
\hline Femenino, 6 años & Pierna derecha & $7 \mathrm{~cm}$ por $1,5 \mathrm{~cm}$ & Escaldadura & 10 meses \\
\hline Femenino, 6 años & Pierna izquierda & $7 \mathrm{~cm}$ por $2 \mathrm{~cm}$ & Escaldadura & 11 meses \\
\hline Masculino, 12 años & Pierna izquierda & $9 \mathrm{~cm}$ por $5 \mathrm{~cm}$ & Quemadura, fuego & 6 meses \\
\hline Femenino, 12 años & Hombro izquierdo & $15 \mathrm{~cm}$ por $12 \mathrm{~cm}$ & Quemadura, fuego & 11 meses \\
\hline Masculino, 16 años & Nariz & $1 \mathrm{~cm}$ por $1 \mathrm{~cm}$ & Quemadura, fuego & 11 meses \\
\hline Masculino, 16 años & Cara y cuello & $12 \mathrm{~cm}$ por $7 \mathrm{~cm}$ & Quemadura, fuego & \\
\hline
\end{tabular}

hasta completar 6 sesiones. No observamos efectos secundarios o colaterales con la administración del fármaco.

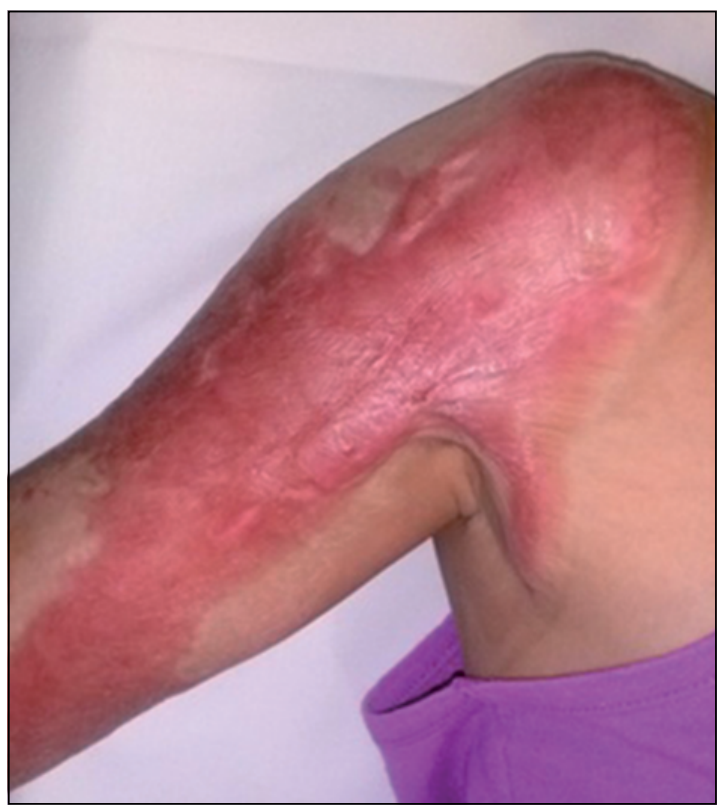

Figura 1 Cicatriz patológica antes del tratamiento ejemplo 1.
Las Tablas 2 y 3 muestran los resultados que comparan antes y después del tratamiento con escalas Posas y Vancouver.

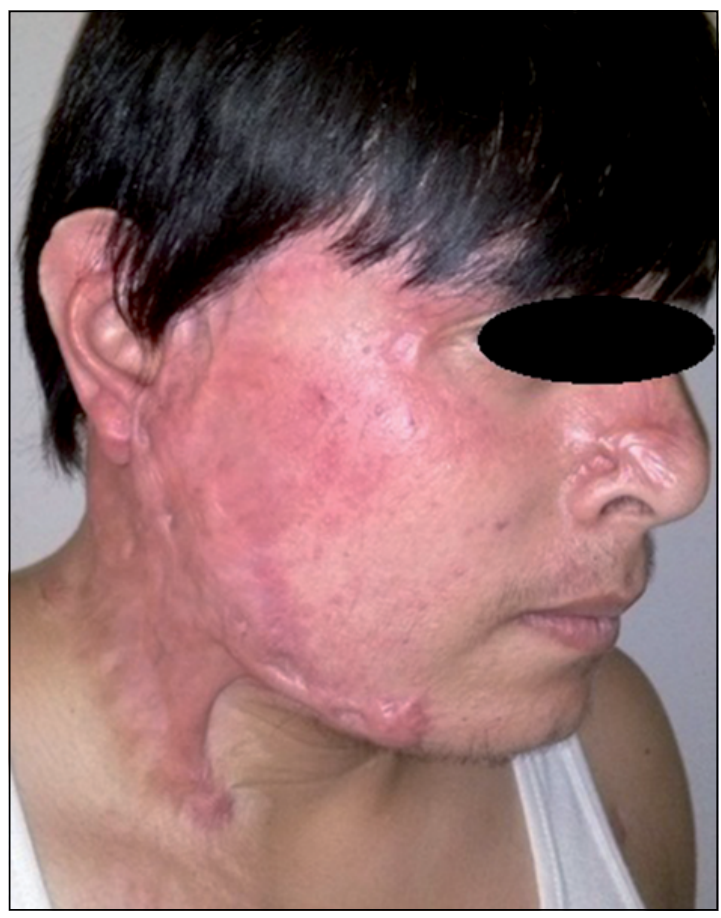

Figura 2. Cicatriz patológica antes del tratamiento ejemplo 2. 


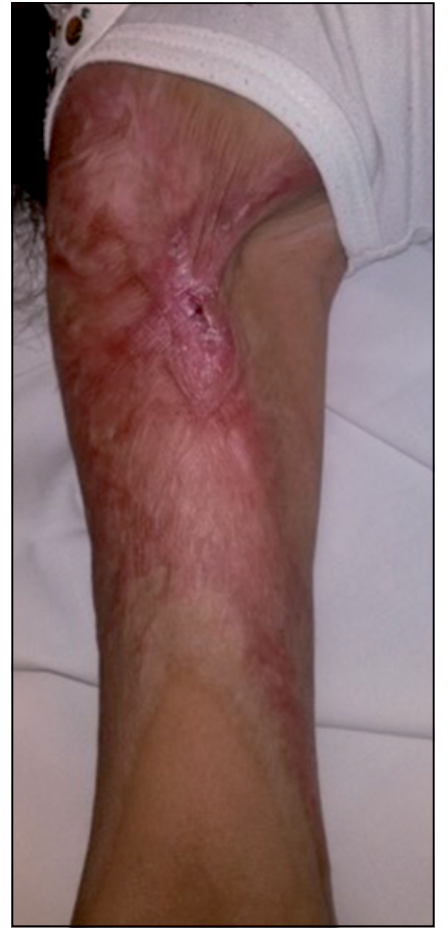

Figura 3. Cicatriz patológica después del tratamiento ejemplo 1 .

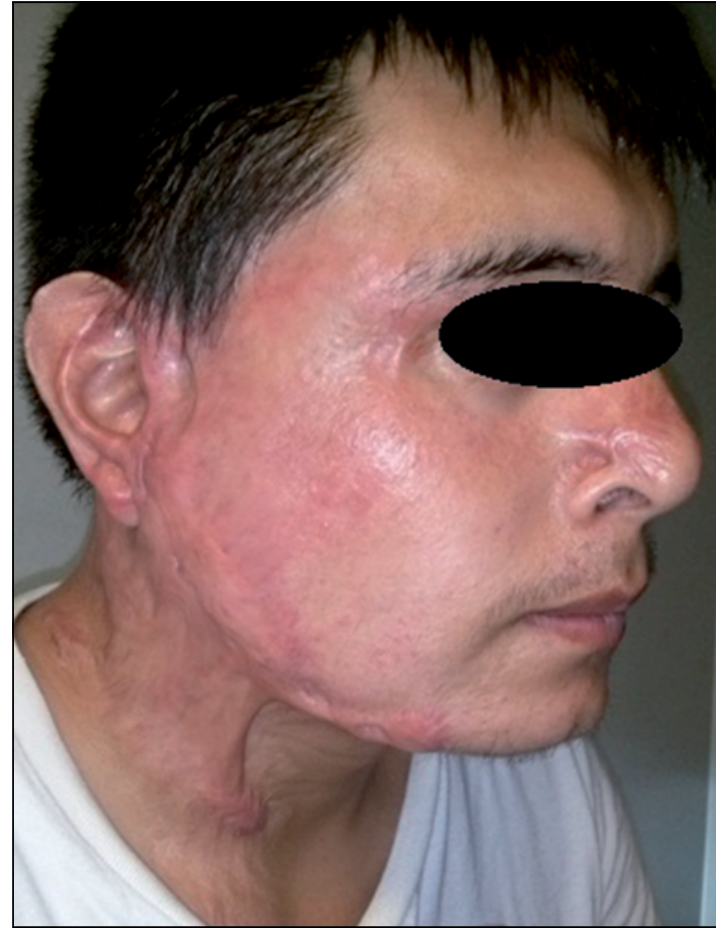

Figura 4. Cicatriz patológica después del tratamiento ejemplo 2.

Tabla 2. Escala de Posas antes y después del tratamiento, se consideró estadísticamente significativo una p igual o menor a 0,05

\begin{tabular}{|c|c|c|c|}
\hline Parámetro & $\begin{array}{c}\text { Promedio y DS } \\
\text { antes del tratamiento }\end{array}$ & $\begin{array}{c}\text { Promedio y DS después del } \\
\text { tratamietno }\end{array}$ & $\mathrm{p}$ igual o menor a 0,05 \\
\hline Vascularidad & $8,1 \pm 2,4$ & $5 \pm 2,76$ & 0,01 \\
\hline Pigmentación & $6,23 \pm 3,11$ & $3,53 \pm 1,61$ & 0,01 \\
\hline Grosor & $6,7 \pm 2,58$ & $4,15 \pm 1,67$ & 0,005 \\
\hline Alivio síntomas & $4,83 \pm 3,56$ & $4 \pm 2,44$ & 0,003 \\
\hline Pliabilidad & $6,23 \pm 3,1$ & $3,53 \pm 1,61$ & 0,01 \\
\hline Irregular & $7,6 \pm 3,1$ & $5,7 \pm 2,27$ & 0,004 \\
\hline
\end{tabular}

Tabla 3. Escala de Vancouver antes y después del tratamiento, se consideró estadísticamente significativo una p menor o igual a 0,05

\begin{tabular}{|lccc|}
\hline Parámetro & $\begin{array}{c}\text { Promedio y DS antes del } \\
\text { tratamiento }\end{array}$ & $\begin{array}{c}\text { Promedio y DS después del } \\
\text { tratamiento }\end{array}$ & p igual o menor a 0,05 \\
\hline Pigmentación & $1,84 \pm 0,37$ & $1,38 \pm 0,5$ & 0,01 \\
\hline Pliabilidad & $3,38 \pm 1,6$ & $1,92 \pm 1,18$ & 0,01 \\
\hline Vascularidad & $1,83 \pm 0,68$ & $1,23 \pm 0,59$ & 0,02 \\
Altura & $1,91 \pm 0,7$ & $1,15 \pm 0,8$ & 0,008 \\
\hline
\end{tabular}




\section{Discusión}

El uso temprano de las prendas de compresión se recomienda de forma rutinaria en diferentes centros alrededor del mundo; si bien no es perjudicial para los pacientes, los informes y las pruebas muestran resultados diferentes. La terapia de presión fue popularizada por primera vez en el Shriners Burn Hospital de Galvestone. En 1971 Larson, del mismo grupo, informó que la terapia de presión disminuyó la cicatrización hipertrófica después de la lesión térmica $^{1}$. Y muchos informes fueron seguidos sin evidencia de estudios controlados. En los últimos años se han publicado diferentes trabajos. Los resultados de estos estudios muestran resultados diferentes. Anzarut y cols., publicaron en 2009, un metaanálisis, que incluye diferentes ensayos que reportan el uso de las prendas de compresión, llegaron a la conclusión de que no hay pruebas suficientes para apoyar el uso habitual de estas mismas ${ }^{2}$.

Las prendas de compresión pueden mejorar el proceso de maduración mediante la restricción del flujo de sangre a la zona de la cicatriz y la compresión constante que inhibe el crecimiento de tejido hipertrófico. La presión facilita el control de la síntesis de colágeno por la reducción del suministro de sangre, oxígeno y nutrientes y se puede notar por la disminución del enrojecimiento de la cicatriz y el edema ${ }^{1,2}$.

LH Engrav y su grupo en Seattle hicieron un estudio bien diseñado donde propone un uso racional de las prendas de compresión. El tamaño de la cicatriz, la ubicación y grado de profundidad de la quemadura fueron analizados. Presentaron un estudio retrospectivo durante 12 años con un seguimiento de un año. Se incluyeron 54 pacientes. Llegaron a la conclusión de que el beneficio de esta terapia puede sugerirse en cicatrices por quemaduras de segundo grado profundo y tercer grado en las que se usó injerto de piel, cicatrices que han curado espontáneamente en cuestión de varias semanas, niños y adultos jóvenes, personas de color y lugares del cuerpo donde la compresión se puede aplicar ${ }^{3}$.

En nuestro centro como en muchos centros de todo el mundo, diferentes especialistas están involucrados en el seguimiento de los pacientes. Se promueve el movimiento precoz y la fisioterapia. E1 ultrasonido y la estimulación eléctrica se utilizan de manera habitual cuando la ubicación de la cicatriz lo permite.

El departamento de sicología evalúa cada paciente y propone el tratamiento de acuerdo a diferentes factores, donde la percepción de la cicatriz y la función son el foco principal. El apoyo familiar es importante en el cuidado de nuestros pacientes, con el fin de tener un buen cumplimiento del trata- miento, principalmente en el caso de la terapia de presión, debido a la edad de nuestro paciente se hace difícil de seguir las instrucciones sin la ayuda de los padres. Además de nuestros esfuerzos en algunos casos no se encuentra mejoría de las cicatrices lo cual repercute en la calidad de vida de nuestros pacientes. Teniendo en cuenta la evidencia reciente con el uso de verapamilo propusimos el uso temprano de este medicamento.

Convencidos de que la terapia de presión es una herramienta útil en el tratamiento de la cicatriz después de una lesión por quemadura. Es importante mencionar que no se pueden obtener conclusiones absolutas de este trabajo ya que es esperable que el proceso de maduración de la cicatriz se complete por sí mismo aunque nuestra intención es disminuir el tiempo de maduración mejorando la calidad de vida $\mathrm{y}$ función de nuestros pacientes.

El verapamilo tiene diferentes beneficios sobre el crecimiento queloide del fibroblasto. Hay informes que mostraron el efecto sobre la interleucina 6 (IL 6) y la secreción del factor de crecimiento endotelial vascular, así como en el crecimiento celular en cultivo primario de fibroblastos derivados de la parte central del queloide ${ }^{5,6}$. También tiene efecto en la modulación de la biosíntesis de colágeno, cambia la morfología de los fibroblastos, la reorganización de filamentos de actina, tal como se revela por la tinción con isotiocianato de fluoresceína FITC en el microscopio ${ }^{7,8}$.

El verapamilo solo o en combinación con la cirugía ha mostrado eficacia en el tratamiento de la cicatrización queloide ${ }^{9,10}$.

Recientemente se informó de estudios que comparan verapamilo y triamcinolona. Margaret Shanti y Ernest Dhanraj reportaron un estudio aleatorio, simple ciego donde incluyeron 54 pacientes en los que se encontró la reducción de la vascularización, flexibilidad, altura y anchura de la cicatriz después de 3 semanas de tratamiento. Sin embargo, el resultado fue más rápido en el grupo de triamcinolona; por otro lado, más efectos adversos fueron encontrados con este grupo como hipocromías y fragilidad de la piel ${ }^{11}$.

En otro estudio también en la India, Rajeev Ahuja y Pallab Chartterjee compararon en cicatrices hipertróficas y queloides el uso de verapamilo y triamcinolona. Ellos mostraron en un estudio ciego aleatorio los efectos de ambos medicamentos. Se incluyeron cuarenta pacientes. En 22 cicatrices se usó triamcinolona y en 26 verapamil. Encontraron mejoría en la vascularización de la cicatriz, la flexibilidad y la altura. Propusieron el uso de verapamilo porque el costo es más barato y los efectos adversos son menores ${ }^{12}$. En este estudio se administraron los medicamentos cada 3 semanas. La ventaja del vera- 
pamilo es que puede ser administrado cada 7 a 10 semanas, dando las posibilidades para completar en menos tiempo el esquema de tratamiento.

Sabemos que estudios bien diseñados son necesarios para responder a las preguntas como dosis y posibles efectos secundarios, que probablemente, es una debilidad importante de nuestro informe, sin embargo, estamos llevando a cabo un estudio aleatorio que puede ayudar a aclarar estas preguntas. La mayor parte de la evidencia muestra beneficio con el uso de verapamilo, sin embargo, el tamaño de la cicatriz no es tan grande ${ }^{13}$. Con este reporte nosotros proponemos el uso de verapamilo para ayudar la adherencia al tratamiento con las prendas con compresión en la cicatriz patológica causada por la quemadura en la que inicialmente no se observa mejoría alguna.

\section{Conclusión}

El verapamilo en combinación con terapia de presión mejora las escalas Posas y Vancouver en cicatrices patológicas (queloide y la cicatriz hipertrófica) con antecedente de lesiones por quemaduras. Nuestros pacientes y familiares (padres) refieren mejoría en el prurito, pigmentación, espesor y flexibilidad. Así mismo observamos mejoría en la pigmentación, la flexibilidad, la vascularización y la altura de la cicatriz. Más estudios son necesarios para determinar la dosis ideal de este medicamento en el caso de la administración intralesional en la cicatriz patológica en pacientes con quemaduras.

\section{Referencias}

1. Atiyeh BS, El Khatib AM, Dibo SA. Pressure garment therapy of Burn Scars Evidence Based Efficacy. Annals of Burns and Fire Disaster 2013;26:205-12.

2. Anzarut A, Olson J, Singh P. The effectivenes of pres- sure garment therapy for the prevention of abnormal scarring after burn injury: A meta-analysis. J Plast Reconstr Aesthetic Surg, 2009;62:77-84.

3. Engrav LH, Heimbach DM, Rivara FP. 12 years within wound study of the effectiveness of custom pressure garment therapy. Burns 2010;36:975-83.

4. Boggio RF, Freitas VM, Cassiola FM. Effect of a calcium-channel blocker (verapamil) on the morphology, cytoskeleton and collagenase activity of human skin fibroblasts. Burns 2011;37:616-25.

5. Lee RC, Ping JA. Calcium antagonists retard extracellular matrix production in connective tissue equivalent. J Surg Res. 1990;49:463-6.

6. Doong H, Dissanayake S, Gowrishankar TR. Calcium antagonist alter cell shape and induce procollagenase synthesis in keloid and normal human dermal fibroblasts. J Burn Care Rehabil. 1996;17:497-514.

7. Giugliano G, Pasquali D, Notaro A. Verapamil inhibits intereukin-6 and vascular endothelial growth factor production in primary cultures of keloid fibroblast. $\mathrm{Br} \mathrm{J}$ Plast Surg. 2003;56:804-9.

8. Copcu E, Sivrioglu N, Oztan Y. Combination of surgery and intralesional verapamil injection in the treatment of the keloid. J Burn Care Rehabil. 2004;25:1-17.

9. D'Andrea F, Brongo S, Ferraro G, Baroni A. Prevention and treatment of keloids with intralesional verapamil. Dermatology 2002;204:60-62.

10. Lawrence WT. Treatment of earlobe keloids with surgery plus, adjuvant intralesional verapamil and pressure earrings. Ann Plast Surg. 1996;37:167-9.

11. Ahuja RB, Chatterje P. Comparative efficacy of intralesional verapamil hydrochloride and triamcinolone acetonide in hypertrophic scars and keloids. Burns 2014;40:583-8.

12. Shanti M, Dhanrah P. Comparison of intralesional verapamil with intralesional triamcinolone in the treatment of hypertrophic scars and keloids. Indian J Dermatol Venereol Leprol. 2008;74:343-8.

13. Ledon JA, Savas J, Franca K. Intralesional treatment for keloids and hypertrophic scars: a review. Dermatol Surg. 2013;39:1745-57. 